\title{
Illusory resizing of the painful knee is analgesic in symptomatic knee osteoarthritis
}

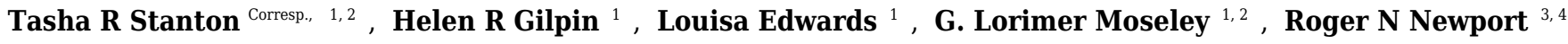 \\ ${ }^{1}$ School of Health Sciences, University of South Australia, Adelaide, South Australia, Australia \\ 2 Neuroscience Research Australia, Randwick, Australia \\ 3 School of Psychology, University of Nottingham, Nottingham, United Kingdom \\ ${ }^{4}$ School of Sport, Exercise and Health Sciences, Loughborough University, Loughborough, United Kingdom \\ Corresponding Author: Tasha R Stanton \\ Email address: tasha.stanton@unisa.edu.au
}

Background: Experimental and clinical evidence support a link between body representations and pain. This proof-of-concept study in people with painful knee osteoarthritis (OA) aimed to determine if: i) visuotactile illusions that manipulate perceived knee size are analgesic; ii) cumulative analgesic effects occur with sustained or repeated illusions.

Methods: Participants with knee OA underwent 8 conditions (order randomised): stretch and shrink visuotactile (congruent) illusions and corresponding visual, tactile and incongruent control conditions. Knee pain intensity (0-100 numerical rating scale; $0=$ no pain at all and $100=$ worst pain imaginable) was assessed pre- and post-condition. Condition (visuotactile illusion vs control) x Time (pre-/postcondition) repeated measure ANOVAs evaluated the effect on pain. In each participant, the most beneficial illusion was sustained for 3 minutes and was repeated 10 times (each during 2 sessions); paired t-tests compared pain at time 0 and 180s (sustained) and between illusion 1 and illusion 10 (repeated).

Results: Visuotactile illusions decreased pain by an average of 7.8 points ( $95 \% \mathrm{Cl} 2.0$ to 13.5 ) which corresponds to a $25 \%$ reduction, but the tactile only and visual only control conditions did not (Condition $x$ Time interaction: $p=0.028$ ). Visuotactile illusions did not differ from incongruent control conditions where the same visual manipulation occurred, but did differ when only the same tactile input was applied. Sustained illusions prolonged analgesia, but did not increase it. Repeated illusions increased the analgesic effect with an average pain decrease of 20 points ( $95 \% \mathrm{Cl} 6.9$ to 33.1) - corresponding to a $40 \%$ pain reduction.

Discussion: Visuotactile illusions are analgesic in people with knee OA. Our results suggest that visual input plays a critical role in pain relief, but that analgesia requires multisensory input. That visual and tactile input is needed for analgesia, supports multisensory modulation processes as a possible explanatory mechanism. Further research exploring the neural underpinnings of these visuotactile illusions is needed. For potential clinical applications, future research using a greater dosage in larger samples is warranted. 
Illusory resizing of the painful knee is analgesic in symptomatic knee osteoarthritis

${ }^{1}$ School of Health Sciences \& PainAdelaide Consortium, The University of South Australia,

Adelaide, SA, Australia

${ }^{2}$ Neuroscience Research Australia, Sydney, NSW, Australia

${ }^{3}$ School of Psychology, The University of Nottingham, Nottingham, UK

\section{Corresponding Author:}

Tasha R Stanton

21 The University of South Australia

22 School of Health Sciences - CEA-14

23 GPO Box 2471, Adelaide, South Australia 5001

24 Phone: $+61883022090 \quad$ Fax: +61883022853

25 Email: Tasha.stanton@unisa.edu.au 
Abstract:

27 Background: Experimental and clinical evidence support a link between body representations and pain. This proof-of-concept study in people with painful knee osteoarthritis (OA) aimed to determine if: i) visuotactile illusions that manipulate perceived knee size are analgesic; ii) cumulative analgesic effects occur with sustained or repeated illusions.

Methods: Participants with knee OA underwent 8 conditions (order randomised): stretch and shrink visuotactile (congruent) illusions and corresponding visual, tactile and incongruent control conditions. Knee pain intensity $(0-100$ numerical rating scale; $0=$ no pain at all and $100=$ worst pain imaginable) was assessed pre- and post-condition. Condition (visuotactile illusion vs control) x Time (pre-/post-condition) repeated measure ANOVAs evaluated the effect on pain. In each participant, the most beneficial illusion was sustained for 3 minutes and was repeated 10 times (each during 2 sessions); paired t-tests compared pain at time 0 and 180 s (sustained) and between illusion 1 and illusion 10 (repeated).

Results: Visuotactile illusions decreased pain by an average of 7.8 points (95\% CI 2.0 to 13.5 ) which corresponds to a $25 \%$ reduction, but the tactile only and visual only control conditions did not (Condition $\mathrm{x}$ Time interaction: $\mathrm{p}=0.028)$. Visuotactile illusions did not differ from incongruent control conditions where the same visual manipulation occurred, but did differ when only the same tactile input was applied. Sustained illusions prolonged analgesia, but did not increase it. Repeated illusions increased the analgesic effect with an average pain decrease of 20 points $(95 \%$ CI 6.9 to 33.1$)$ - corresponding to a $40 \%$ pain reduction.

Discussion: Visuotactile illusions are analgesic in people with knee OA. Our results suggest that visual input plays a critical role in pain relief, but that analgesia requires multisensory input. That 
48 visual and tactile input is needed for analgesia, supports multisensory modulation processes as a

49 possible explanatory mechanism. Further research exploring the neural underpinnings of these

50 visuotactile illusions is needed. For potential clinical applications, future research using a greater

51 dosage in larger samples is warranted. 
52

53

54

55

56

57

58

59

60

61

62

63

64

65

66

67

68

69

70

71

72

73

74

\section{Introduction:}

Knee osteoarthritis (OA) affects $3 \%$ of the global population (Cross et al. 2014) and is a condition for which treatment is not always straightforward. Given the discordance between the extent of structural damage on imaging and the extent of joint pain (Hannan et al. 2000) as well as the occurrence of severe joint pain after total knee replacement (Wylde et al. 2011), it is acknowledged that other neural factors likely contribute to the pain experienced by those with knee OA.

Recent experimental and clinical research has highlighted intriguing links between body representations and pain (Longo et al. 2009; Longo et al. 2012; Mancini et al. 2011; Moseley et al. 2008; Preston \& Newport 2011). Experimental evidence supports the presence of visually induced analgesia, that is, merely having vision of your own body (versus of an object) reduces pain (Longo et al. 2009). Perceived characteristics of the body also modulate this analgesia, but this effect is not straightforward (Boesch et al. 2016). For example, magnifying the visual input of the hand - making the entire hand appear larger - increases the extent of analgesia in experimental pain (Mancini et al. 2011), but has the opposite effect in pathological hand pain (Moseley et al. 2008) and has no effect in painful hand OA (Preston \& Newport 2011). Rather, in painful hand OA, combining both touch input and visual manipulation (i.e., a visuotactile illusion: visually increasing the size of the hand while also gently pulling on the fingers) to provide a site-specific change in morphology is analgesic (Preston \& Newport 2011).

Specifically, these visuotactile illusions in OA provide a non-affine change in hand morphology. That is, the overall hand does not change size, rather it only 'stretches' from one point. Such an illusion which localises its effect to a specific area seems intuitively relevant for a condition such as OA where pain is usually limited to a specific joint. Intriguingly, in hand OA sometimes the 
analgesic visuotactile manipulation is a stretched looking hand and other times it is a shrunken looking hand (Preston \& Newport 2011), suggesting that the effect might be individually specific.

While some controversy exists regarding the analgesic effects of body illusions (Gilpin et al. 2014; Martini et al. 2014b; Mohan et al. 2012), recent work has highlighted that that differing effects likely relate to differences in methodology (Martini et al. 2014a; Nierula et al. 2017). Indeed, there is a growing body of literature on the theoretical and clinical implications of bodily illusions (Moseley et al. 2012) and our recent systematic review and meta-analysis highlighted their clear therapeutic potential (Boesch et al. 2016). Importantly, that review also identified the variability of results across methods, experimental, and clinical conditions and emphasised the need for more rigorous and controlled experiments in different types of painful conditions (Boesch et al. 2016). The review also found that most studies evaluated only very small dosages (i.e., the effect of only a single illusion intervention) (Boesch et al. 2016). Evaluating potential cumulative benefit of repeated or sustained illusions are key for clinical relevance.

This exploratory proof-of-concept study aimed to determine whether visuotactile illusions are analgesic for people with painful knee OA. We hypothesised that visuotactile illusions would result in a significantly larger pain reduction than observed during control conditions, although we were uncertain whether vision-only bodily illusions may also provide benefit given previous contradictory findings described above. Last, we explored whether sustained or repeated trials might offer cumulative benefit.

\section{Materials \& Methods:}


96 Participants: Participants with current knee pain and a clinical diagnosis of knee OA (Altman et

97 al. 1986) were recruited from the community via newspaper advertisements, recruitment posters,

98 and word of mouth. Specifically, if radiographic evidence of osteoarthritic changes were present,

99 then participants were required to have current knee pain and meet one of the following criteria:

100 age $>50$ years; morning stiffness $<30$ minutes; crepitus of the knee. If radiographs were not

101 available, then participants were required to have current knee pain and meet at least three of the

102 following criteria: age $>50$ years; morning stiffness $<30$ minutes; crepitus of the knee; bony

103 tenderness of the tibiofemoral joint line; bony enlargement of the knee; no palpable warmth.

104 Those with rheumatoid or inflammatory arthritis, with neurological disorders affecting the lower

105 limb, or with cognitive impairment were excluded. All participants provided written, informed

106 consent as per the Declaration of Helsinki. This research was approved by The University of

107 South Australia’s Human Research Ethics Board (Protocol No.: 0000028496).

108 Past work in symptomatic hand OA found large analgesic effects of visuotactile illusions when

109 compared with a control condition (Cohen's $\mathrm{f}=0.6)$ (Preston \& Newport 2011). Using $\mathrm{f}=0.6$,

110 power $=0.80$, alpha $=0.05$, and repeated measures correlation of 0.6 , we would need 6

111 participants to detect similar effects. Using G*Power 3 (Faul et al. 2007), we conservatively

112 powered to detect a moderate-large (Cohen 1969) effect on pain (Cohen's $f=0.35$; equivalent to

113 a partial $\eta^{2}$ of 0.11 ), resulting in a required sample of 12 participants.

114 Equipment: The MIRAGE-mediated reality system (Preston \& Newport 2011) was used to

115 provide two types of visuotactile illusions. One induced a feeling of stretching the knee (stretch

116 illusion) and one induced a feeling of shrinking or compressing the knee (shrink illusion).

117 Illusions were induced with the participant either in sitting or standing. Participants wore a head

118 mounted display that showed a live video feed of their own knee. If tested in sitting, this set-up 
119 allowed them to view their knee and leg from a first-person perspective and in the same spatial

120 location as if they were looking down at their own knee (i.e., camera above and slightly behind

121 their head, pointing downwards at their knee). The non-test limb was draped with a black cloth

122 so that participants could only see their test limb. If tested in standing, participants saw their leg

123 in third-person perspective (i.e., camera in front of the leg), but were advised to imagine that they

124 were looking at their own limb that was reflected in a large mirror placed in front of them.

125 Participants stood surrounded by black sheets hanging from ceiling to floor (on the left side, right

126 side and behind them), with the non-test limb covered using a black cloth such that they only had

127 vision of their test limb. In both set-ups participants were familiarised with the technology and

128 underwent a standardised procedure to promote ownership of the limb, i.e., participants moved

129 their legs, flexed their quadriceps muscle and were touched on the leg ( four minutes in total),

130 all while watching their own leg in the head mounted display video feed. The choice of illusion

131 set-up (sitting or standing) was determined by which of the two postures was associated with the

132 participant's typical knee pain. A customised Labview program (National Instruments 2015;

133 Austin TX) was used to digitally alter the video feed in real-time, such that participants watched

134 their own limb undergo a real-time change in size.

135 The visuotactile illusions used in this study provide temporally and directionally congruent

136 visual and tactile information to create a sense that the body is truly changing in size (See Video

$137 \mathrm{~S} 1$ ). In the stretch illusion, as the video image of the knee was elongated (making the knee joint

138 appear to stretch or grow), the experimenter applied gentle tactile traction to participant's calf

139 muscle (pulling towards the foot) to provide 'directionally congruent' information. Similarly, in

140 the shrink illusion, gentle tactile compression (push towards the knee) was accompanied by

141 visual shrinkage of the knee. These manipulations have been found to alter perceptions of body 
142 size (Gilpin et al. 2015), reduce pain in people with hand OA (Preston \& Newport 2011) and

143 induce the feeling that the knee is actually stretching or shrinking.

144 Procedure: Participants attended three sessions. In Session One, we collected demographic (age,

145 sex, height, weight) and OA-specific information (history of knee pain [years]; minimum,

146 maximum, and average knee pain over the past 48 hours using a 0-100 numerical rating scale

147 [NRS], where $0=$ no pain at all and $100=$ worst pain imaginable). Participants then completed

148 the Oxford Knee Score questionnaire (Dawon et al. 1998) to evaluate knee function and the

149 Fremantle Knee Awareness Questionnaire (Nishigami et al. 2017) to evaluate body perception

150 related to the knee. Participants completed a perceived knee size experimental task, using

151 established methodology (Gilpin et al. 2015). In brief, participants were presented with a visual

152 image of their own knee that was too small (80\%) and too large (120\%). These images were

153 increased and decreased in size, respectively, with participants advised to verbally indicate when

154 the image looked to be the right size of their own knee. The order (small/large) was randomised

155 and the procedure was completed twice for each image size presentation (Gilpin et al. 2015).

156 Following completion of baseline assessment, participants underwent eight conditions in a

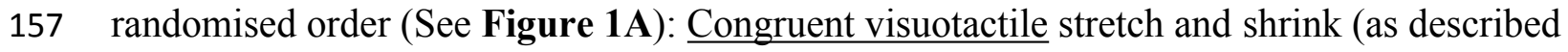

158 above); Vision only stretch and shrink (visual image elongates/shrinks; experimenter's hand on

159 leg but no tactile force provided); Tactile only stretch and shrink (tactile traction/ compression,

160 no visual change); Incongruent visuotactile stretch and shrink (visual stretch, but tactile

161 compression; visual shrink, but tactile traction). For ease of reading, these conditions will be

162 referred to as Congruent VT, VO, TO, and Incongruent VT, respectively. The participants were

163 blinded to condition: no information about the real illusion was provided. Pain intensity, assessed

164 using a 101-point numerical rating scale (where $0=$ no pain at all and $100=$ worst pain 
165 imaginable) was evaluated before and after each condition. Each condition took $\sim 30$ seconds to

166 complete and there was a two minute break between each condition.

167 Following application of the eight conditions, the congruent VT illusion that resulted in the 168 greatest pain reduction immediately post-illusion was then applied for a second time and 169 sustained for three minutes while participants viewed their knee in this altered state. Pain 170 intensity was reported every 30 seconds during this three minute period. The total duration of

171 Session One (including baseline questionnaires; See Table 1) was approximately one hour.

172 Sessions Two and Three (minimum of two weeks apart; maximum of 3 weeks) used the illusion 173 that was determined most analgesic during Session One. In Session Two, the effect of a three 174 minute sustained illusion was evaluated again (assessing pain every 30 seconds). In Sessions 175 Two and Three, ten trials of the illusion were performed, assessing pain intensity pre- and post176 illusion. During testing with the congruent VT illusion, participants were asked whether or not it 177 felt as though the manipulation was occurring to their own leg (yes/no). This question was not 178 asked during the eight conditions of Session One in order to maximise participant blinding to the 179 'real' illusion. Last, participants recorded their average daily pain scores between the second and 180 third session using a pain diary.

181 Statistical analysis: All statistics were performed using IBM SPSS 22.0. Data were assessed for 182 normality (using visual inspection and Shapiro-Wilk statistic) and for sphericity (using 183 Mauchly's test of sphericity). If the normality assumption was not met for raw and for 184 transformed data, non-parametric analyses were used. If the sphericity assumption was violated, 185 Greenhouse-Geissier corrections were applied. 
186 Our pilot data in those with knee OA $(n=3)$ showed that one type of congruent VT illusion (e.g.,

187 stretch) was more analgesic than the other illusion (e.g., shrink) and control conditions; but

188 whether the analgesic illusion was stretch or shrink varied between participants. Thus our

189 analysis plan, determined a priori, identified the congruent VT illusion (stretch or shrink) that

190 was most analgesic in each participant and compared pain ratings with those of the relevant

191 control conditions (See Figure 1B).

192 To determine if the congruent VT illusion provided analgesia above that provided by its

193 component parts (VO, TO) we performed a 2 (Time: pre-/post-condition) x 3 (Condition)

194 repeated measures analysis of variance (RM ANOVA). Post-hoc paired t-tests were used to

195 explore any significant effects, using a Holm-Bonferroni correction (Holm 1979) to control for

196 multiple comparisons. To determine if the congruent nature of visuotactile input was important,

197 we performed a 2 (Time) x 2 (Condition: congruent vs incongruent) RM ANOVA. Separate

198 analyses were completed to compare to each incongruent condition (i.e., vision-controlled and

199 touch-controlled). Additionally, given that frame of reference (first-person versus third-person

200 perspective) has been shown to play a critical role in the phenomenal experience of body

201 ownership and the effectiveness of experiencing bodily illusions (Blanke 2012), we repeated the

202 above analyses, including Perspective (first- vs third-person; relating to whether the participant

203 was seated or standing during testing, respectively) as a between subject factor. Because we did

204 not initially power for this analysis, it was considered exploratory.

205 Given that visual distortion of body size alone (i.e., no tactile component) is analgesic (Mancini

206 et al. 2011), and that varying effects are seen between individuals with chronic pain (Preston \&

207 Newport 2011) we performed a supplementary analysis comparing pain scores (pre-/post- 
208 condition) from the 'best' condition with those for the relevant tactile control condition. The

209 'best' condition was considered the most analgesic of either VO or congruent VT conditions.

210 Last, to determine if sustained illusion or multiple trials offered extra benefit, paired t-tests

211 compared pain intensity: i) immediately post-illusion versus end of three minutes (sustained); ii)

212 the $1^{\text {st }}$ illusion versus the $10^{\text {th }}$ illusion (repeated). To determine if there was a decrease in

213 average daily pain (last 48 hours), paired t-tests compared the baseline measures of average pain

214 with the average pain directly after the $2^{\text {nd }}$ session and prior to the $3^{\text {rd }}$ session (the latter two

215 involving the average of the daily pain scores for two days).

217 Results:

218 Fourteen participants were screened for inclusion; two were ineligible because they did not meet

219 ACR criteria for knee OA (Altman et al. 1986). Both ineligible participants did not have

220 radiographs of their knee and neither satisfied at least three of the six clinical criteria. One

221 participant had knee pain following total knee replacement surgery but was included because it

222 mirrored the original osteoarthritic knee pain. Twelve participants completed Session One; six

223 completed Session Two (three had no pain in sitting/standing, and thus could not be tested; three

224 dropped out due to time commitments and reported difficulties getting to the testing lab); seven

225 completed Session Three (two participants had no pain). During sustained and repeated testing

226 with the congruent VT illusion, all participants reported that it felt as though the manipulation

227 was occurring to their own limb (for both testing set-ups: 6 - sitting; 6 - standing). Full

228 participant demographics are provided in Table 1. All outcomes were normally distributed and 229 thus parametric statistics were used.

230 Effect of congruent VT illusion versus control conditions (Figure 2A). 
231 The congruent VT illusion resulted in significantly more analgesia than the TO and the VO

232 control conditions (Figure 2A). There was no effect of Condition $\left(F_{2,22}=0.93, p=0.41\right.$ ), no

233 effect of Time $\left(\mathrm{F}_{1,11}=4.7, \mathrm{p}=0.053\right)$, but a Condition $\mathrm{x}$ Time interaction $\left(\mathrm{F}_{2,22}=4.2, \mathrm{p}=0.028\right)$.

234 Paired t-tests showed no change in pain during the TO $\left(\mathrm{t}_{1,11}=1.45, \mathrm{p}=0.17\right)$ and VO control

235 conditions $\left(\mathrm{t}_{1,11}=-0.71, \mathrm{p}=0.95\right)$, but a significant pain reduction during the congruent VT

236 illusion $\left(\mathrm{t}_{1,11}=2.96, \mathrm{p}=0.013\right)$. Pain decreased by an average of 7.8 points (95\% CI 2.0 to 13.5$)$,

237 corresponding to a $25 \%$ reduction from pre-illusion pain scores. Considering perspective type

238 (first- vs third-person) in the analysis did not change the findings and there was no main effect of

239 Perspective or any of its interactions (See Supplementary File 1).

240 The congruent VT illusion pain ratings did not differ from the incongruent VT condition that

241 controlled for vision (Figure 2B). That is, when identical visual manipulation occurred, there

242 was a main effect of Time $\left(\mathrm{F}_{1,11}=12.6, \mathrm{p}=0.005\right)$, but no effect of Condition $\left(\mathrm{F}_{1,11}=0.032, \mathrm{p}=\right.$

243 0.86), or Condition $\mathrm{x}$ Time interaction $\left(\mathrm{F}_{1,11}=0.34, \mathrm{p}=0.57\right)$, suggesting that analgesia was

244 provided by both conditions. These findings were unchanged when considering first- versus

245 third-person perspective and there was no main effect of Perspective or any of its interactions

246 (See Supplementary File 1). In contrast, the congruent VT illusion resulted in significantly more

247 analgesia than the incongruent VT condition that controlled for tactile input. That is, when

248 identical tactile input occurred, there was no effect of Condition $\left(F_{1,11}=0.73, p=0.41\right)$, and a

249 main effect of Time $\left(\mathrm{F}_{1,11}=5.23, \mathrm{p}=0.043\right)$, driven by a Condition $\mathrm{x}$ Time interaction $\left(\mathrm{F}_{1,11}=\right.$

$2505.29, \mathrm{p}=0.042$ ) whereby the incongruent VT condition (touch controlled) did not result in

251 analgesia $\left(t_{1,11}=1.26, p=0.23\right)$. Again, these findings were largely unchanged when considering

252 first- vs third-person perspective, the only difference found was that the Condition $\mathrm{x}$ Time 
253 interaction became non-significant $(p=0.052)$, although this is likely due to reduced power (See

254 Supplementary File 1).

255 The exploratory analysis comparing the 'best' condition (congruent VT or VO) to the TO control 256 condition found similar findings (no effect of Condition, $F_{1,11}=1.10, p=0.32$; main effect of

257 Time, $\mathrm{F}_{1,11}=14.4, \mathrm{p}=0.002$; Condition $\mathrm{x}$ Time interaction, $\left.\mathrm{F}_{1,11}=10.6, \mathrm{p}=0.008\right)$, but enhanced

258 analgesia. Paired t-tests showed a significant reduction in pain for the 'best' illusion $\left(\mathrm{t}_{1,11}=4.2, \mathrm{p}\right.$

$259=0.002)$, with an average pain reduction of 11.9 points $(95 \%$ CI 5.6 to 18.2$)$, corresponding to a

$26037 \%$ reduction in pain. There was no change in pain for the TO condition $\left(\mathrm{t}_{1,11}=1.5, \mathrm{p}=0.17\right)$.

261 Effect of sustained illusions on pain (Table 1).

262 There was no additional analgesic effect of sustained viewing of the congruent VT illusion, but

263 the initial effect was sustained. Pain intensity immediately after the illusion did not differ from

264 pain intensity after 3 minutes of sustained viewing of the illusion (Session $1: t_{1,10}=0.52, p=$

265 0.61; Session 3: $\left.\mathrm{t}_{1,7}=-0.697, \mathrm{p}=0.51\right)$.

266 Effect of repeated illusions on pain

267 In Session Two, pain scores for congruent VT illusion one did not differ from illusion ten (pre-

268 illusion scores: $\mathrm{t}_{1,5}=1.4, \mathrm{p}=0.21$; post-illusion scores: $\mathrm{t}_{1,5}=1.1, \mathrm{p}=0.33$ ). However, in Session

269 Three pain scores following illusion ten were significantly reduced compared with pain scores

270 for illusion one (pre-illusion: $\mathrm{t}_{1,6}=3.5, \mathrm{p}=0.013$; post-illusion: $\mathrm{t}_{1,6}=3.9, \mathrm{p}=0.008$; Figure 3).

271 The analgesic effect was large: a reduction of 20 points $(95 \%$ CI 6.9 to 33.1$)$ from the $1^{\text {st }}$ to $10^{\text {th }}$

272 illusion, corresponding to a $40 \%$ reduction in pain.

273 Effect of illusions on daily pain scores (Table 1). 
274 There was no difference between average knee pain (last 48 hours) at baseline and average daily

275 pain in the 48 hours after Session Two $\left(t_{1,6}=0.54, p=0.61\right)$ or the 48 hours prior to Session Three

$276\left(\mathrm{t}_{1,6}=-1.31, \mathrm{p}=0.24\right)$. However, average daily pain scores (last 48 hours) were significantly lower

277 directly after Session Two than those taken just prior to Session Three $\left(\mathrm{t}_{1,6}=2.70, \mathrm{p}=0.036\right)$.

\section{Discussion:}

279 We found evidence that illusory knee resizing using visuotactile manipulation is analgesic in 280 people with osteoarthritic knee pain. Congruent VT illusions reduced pain, while the individual

281 touch and vision components did not, suggesting that pairing of sensory input is important to the

282 analgesic effect. Contrary to our hypothesis, whether or not the tactile input 'directionally

283 matched' the visual input appeared less important. Congruent VT illusions were not more

284 effective at reducing pain than incongruent VT conditions that involved identical visual input

285 (but opposite tactile input), but were more effective than incongruent conditions that involved

286 identical tactile input (and opposite visual input). This suggests that vision is critical to the

287 effect, but requires the pairing of multisensory input (i.e., tactile) to alter pain. Last, prolonged

288 viewing of the illusion sustained analgesia, but did not increase its magnitude. Repeated

289 application of these illusions increased analgesia, but may require a larger dosage than ten

290 illusions to achieve the added benefit. Daily pain scores were not affected by this brief

291 experimental dosage.

292 Analgesic differences between congruent VT illusions and the visual and tactile components

293 Our results in those with knee OA support past work showing that bodily illusions can modulate

294 clinical pain (Boesch et al. 2016). That congruent VT illusions were analgesic and that separate

295 visual and tactile components were not, suggests the presence of a super-additive effect on pain 
296 during the VT illusion. Such effects are the hallmark of multisensory integration, classically

297 demonstrated by behavioural and perceptual responses that exponentially improve with

298 multisensory versus unisensory input (Stein \& Stanford 2008). Greater analgesia with congruent

299 VT illusions than tactile input alone (TO condition) suggests that changes in nociceptive drive

300 (via traction/pressure changes) or gating at the spinal cord via tactile input (Kakigi \& Watanabe

301 1996), are unlikely to contribute to the effect observed. Vision of the body and visual resizing of

302 the body has analgesic effects in experimental pain (Longo et al. 2009; Longo et al. 2012), but,

303 consistent with findings in hand OA (Preston \& Newport 2011), we did not see such an effect

304 here.

305 Comparison of analgesic effects between types of illusion (affine versus non-affine illusion)

306 It is also interesting to consider the impact of the type of illusion provided - that is, whether it

307 was affine (i.e., a rigid body transformation that magnified or minimized the entire body part) or

308 non-affine (i.e., a non-rigid body transformation that altered only part of the body part). Past

309 work has shown that visual illusions that magnify the overall size of the hand (i.e., affine

310 illusions) have contradictory effects on pain dependent upon the condition. For example,

311 magnifying the hand reduces experimental pain (Mancini et al. 2011), but increases pain in those

312 with pathological limb pain (Moseley et al. 2008), and has no effect in people with painful hand

313 OA (Preston \& Newport 2011). However, non-affine alterations that provide site-specific visual

314 morphology changes (e.g., stretch or shrink illusions) are analgesic in hand OA (Preston \&

315 Newport 2011). The present work shows that non-affine alterations to the knee are also analgesic

316 in people with knee OA. Further it extends past work by showing that non-affine visual only

317 change (no tactile component) does not modulate pain. Given past findings of no effect on pain

318 of overall hand size visual change (affine) in people with hand OA (Preston \& Newport 2011), 
319 our results support the view that the type of illusion (affine vs non-affine) and the components of

320 the illusion (i.e., visuotactile) are key to the analgesic effect in painful OA.

321 Differing amounts of analgesia induced by illusion for hand and knee OA

322 That congruent VT illusions provide analgesic benefit in knee OA is consistent with findings in

323 hand OA (Preston \& Newport 2011); however, the magnitude of effect seen here was not as

324 large ( $25 \%$ vs $45 \%$ pain reduction, respectively). There are several potential explanations for this

325 difference. First, various studies show that tactile input from the hand is more precisely

326 represented in the primary somatosensory cortex (S1) than tactile input from the knee (Catley et

327 al. 2013; Mancini et al. 2014; Penfield \& Boldrey 1937). Given that body resizing illusions are

328 thought to target brain-held body maps (see (Schaefer et al. 2007) for evidence of S1 changes

329 with altered visual input of arm size), this less precise cortical representation of the knee might at

330 least partly explain the differing responses to VT illusions and therefore the size of the analgesic

331 effect.

332 Second, it may be that body-specific multimodal integration of vision and touch (a hypothesised

333 mechanism, via S1 inhibition (Cardini et al. 2014), for pain modulatory effects of visuotactile

334 illusions), that occurs in the superior colliculus (Stein et al. 2014), the premotor area and the

335 posterior parietal cortex (PPC) (Avillac et al. 2004; Bremmer et al. 2001), may differ based on

336 bodily site. Studies of multisensory illusions show fundamental differences in the process of

337 multisensory integration in the lower versus upper limbs, with the legs appearing less sensitive to

338 sensory inputs (Pozeg et al. 2015; van Elk et al. 2013). Further, that we spend a great deal of

339 time throughout our development watching our hands closely as we manipulate objects, would

340 suggest that visuotactile representations of the hands may be more efficacious and sensitive than 
341 those of the knee. Together these findings would support a reduced analgesic effect in the knee

342 versus the hand.

343 Third, illusory resizing inherently results in a spatial incongruence between the visually

344 perceived size, and the actual size, of the body part. The impact of this incongruence on pain

345 may differ between the hand and the knee. In the hand, incongruence between body-specific

346 information and spatial information (i.e., crossing the hands over midline) is analgesic, and this

347 effect occurs in later stages of processing of the nociceptive signal, which is thought to coincide

348 with integration of body relevant information in the PPC (Gallace et al. 2011). Such

349 incongruence may impair multisensory processing, thus modulating pain. However, in the lower

350 limb, multisensory integration is not modulated by limb crossing (van Elk et al. 2013), therefore

351 it is possible that analgesic effects induced by impairments in multisensory processing are not

352 present.

353 Spatial incongruence of viewed and actual body size as an analgesic mechanism for body

354 illusions is not straightforward. Many people with chronic pain have been shown to have

355 distorted perceptions of the size of their painful body part (Lewis \& Schweinhardt 2012;

356 Moseley 2005) (see (Moseley et al. 2012) for review) - including those with OA (Gilpin et al.

357 2015; Nishigami et al. 2017). Incongruence between predicted and actual movement (heightened

358 by inaccurate perceptions of the body) may be algesic in some conditions (McCabe et al. 2007;

359 McCabe et al. 2003), although see also (Moseley \& Gandevia 2005). It is interesting to consider

360 whether illusions may normalise pain-induced distortions in bodily size perception - that is, does

361 changing the perceived size of the knee actually reduce body-specific incongruence because the

362 brain-held perception of its size is already inaccurate? On average, participants overestimated the

363 actual size of their knee (Table 1; perceived knee size). This might suggest that they would 
364 prefer a shrink illusion: if mental representation is too large then showing a smaller knee to

365 shrink the representation should be best (i.e., visually normalising size). However, one may

366 argue that if a body part is perceived as being too big, then an illusion that matches the visual

367 size that the body is expected to be may be analgesic (i.e., visually matching expected size).

368 Indeed, most participants responded to illusory stretch (only 3 to illusory shrink), which may

369 support the latter idea. Regardless, that most participants responded to illusory stretch (despite

370 overestimating their knee size) may not be inconsistent with a hypothesis of normalising body

371 perception. Past work has shown that regardless of the type of illusory manipulation (stretch or

372 shrink), distortions in perceived hand size in people with hand OA normalise to that of hand size

373 chosen by healthy pain-free volunteers (Gilpin et al. 2015). Clearly further work is needed to

374 disentangle such effects.

375 Importance of visuotactile input, but not directional congruence

376 That incongruent and congruent VT illusions provided equivocal analgesic effects, but only

377 when the same visual manipulation occurs, suggests that visual input is critical. But visual input

378 alone (i.e., VO) is not sufficient to produce analgesia, highlighting that multisensory input (i.e.,

379 tactile) is required to influence pain. Why might this be? It is possible that inclusion of tactile

380 input (regardless of directional congruence with vision) increases the sense of ownership - the

381 feeling that this is happening to 'my leg'. Experimental pain models support that increases in

382 ownership (of a rubber hand) are analgesic (Siedlecka et al. 2014). However, this hypothesis

383 remains speculative given that we did not formally evaluate ownership for each of the

384 experimental conditions in Session One.

385 Effects on multisensory integration in the lower limb may also occur without the need for

386 congruent tactile input. For example, having a first-person viewpoint during lower limb illusions 
387 (i.e., congruent vision and proprioceptive input) increases visuotactile integration, but congruent

388 tactile input (i.e., synchronous vs asynchronous tapping) does not provide an additional effect

389 (van Elk et al. 2013). While some participants had a third-person viewpoint during illusions, our

390 supplementary analysis evaluating the effect of viewpoint on pain ratings, showed no effect of

391 first- versus third-person perspective. While this analysis may be underpowered to detect such an

392 effect, it may also be that our strategy of having participants imagine that they had a large mirror

393 in front of them (and thus were viewing a mirrored image of their limb), resulted in assuming a

394 first-person perspective. Past work has shown that perceiving a mannequin as mirrored when

395 viewing it in a third-person perspective results in similar levels of ownership as when viewing

396 the mannequin in a first-person perspective (Preston et al. 2015).

397 Last, perhaps vision overrides tactile input. Given that vision provides us with (usually) reliable

398 and precise sensory information about our body, it may be that increased precision of visual

399 input is sufficient to dominate direction information from tactile input (i.e., we do not detect

400 directional incongruence). Our work shows that vision is heavily weighted when judging the

401 location of our body (i.e., the hand), even when proprioception provides contradicting, and

402 accurate, information (Bellan et al. 2015). Past work has shown that incongruent VT input (using

403 the rubber hand/full body illusion set-up) does not induce an illusory percept and is not

404 associated with strong multimodal integration due to spatiotemporal incongruence between

405 visual and tactile input (Blanke et al. 2015). Thus it may be argued that multimodal integration

406 and the generation of an illusory percept does not underlie the analgesic effects seen here given

407 that the incongruent VT condition also provided analgesia. However, it is important to note that

408 asynchronous simulation can induce ownership during the rubber hand illusion in some people

409 (albeit not as strongly as synchronous stimulation) (Botan et al. 2018; Rohde et al. 2011) and that 
410 the sense of ownership in multisensory illusions is additionally sensitive to cues about visual

411 appearance and spatial location when those illusions are applied to one's own body (Ratcliffe \&

412 Newport 2017). Further, the present incongruent VT condition may be argued to have spatial

413 congruence (the hand is located and tactile input occurs at the visually seen location on the leg)

414 and temporal congruence (tactile input occurs at the same time the visual change occurs), but not

415 tactile directional congruence (the tactile input does not match the direction of visual change).

416 Given some level of spatiotemporal congruence, this then may allow visual input to take priority

417 and multimodal integration to occur despite some level of directional incongruence. Future work

418 is clearly needed to delineate the mechanism by which this analgesic effect occurs.

\section{Sustained versus repeated illusions}

420 That sustained illusions did not increase analgesic benefit, but that repeated illusions did,

421 suggests that the analgesic effect may be driven by neural processing initiated with viewing the

422 real-time change in body size. Motion is known to capture visual attention (Abrams \& Christ

423 2003); it is possible that this could be one explanation repeated moving illusions having larger

424 analgesic effects than sustained, static illusions. However, static images of magnified hands are

425 analgesic in experimental pain (Mancini et al. 2011) suggesting that analgesic effects are not

426 solely due to motion in the illusion (and may explain why sustained viewing of the illusion

427 sustained analgesia). It is also possible that relative imprecision in visual representations of the

428 knee may result in participants rapidly adopting the sustained illusion as being an accurate

429 reflection of their own knee, thus not triggering additional analgesic effect as the condition is

430 maintained. Unsolicited comments from participants support this - many remarked that during

431 the sustained illusion, they no longer felt that their knee was resized. On the contrary, when 
432 illusions are repeated, cueing of a change in knee size is repeatedly provided, thus potentially re-

433 instating modulatory processes driven by vision.

434 Study limitations:

435 Our study recruited a small sample but conservative, a priori power calculations based on past 436 work (Preston \& Newport 2011), suggest that it was adequately powered. Sessions Two and 437 Three had lower participant numbers, however, this is unlikely to affect the results - sustained 438 illusions were completed in the full sample in Session One with identical findings to that of 439 Session Two and the effect of repeated illusions on pain was large, and significant, in Session 440 Three. While the effects of VT illusions on pain were small ( $\sim 8$ points on a 101-point NRS), 441 these relate to a single five-second illusion; repeated illusions resulted in pain relief of 20 points, 442 which notably meets recommendations for a clinically important difference (Farrar et al. 2001; 443 Salaffi et al. 2004).

444 In our analysis of daily pain scores, we compared a retrospective recall at baseline (i.e., average 445 pain over the last 48 hours) to the mean of two days of average daily pain scores taken post-

446 Session Two and pre-Session Three. Past work has shown good agreement between the recall of 447 average pain over the last 2-7 days and daily pain scores in OA (Nguyen et al. 2014; Perro et al. 448 2011), with low levels of error (Giske et al. 2010), providing confidence in our findings.

449 However, this is a potential limitation. We do note that daily pain ratings were assessed 450 identically between Session Two and Three. Further research on the effect of these illusions on 451 daily pain scores is warranted.

452 A final limitation is that we did not take ratings of the vividness of the congruent VT illusion 453 (and control conditions) during application or of the general ownership that participants had of 
454 the viewed limb during each condition. This was a pragmatic decision made to maximise

455 participant blinding for condition (e.g., participants may be more aware of the 'real' illusion if

456 we cued them to these features). We informally evaluated ownership of the VT illusion that was

457 tested during sustained and repeated illusions (Sessions One - Three) by asking participants if

458 what they experienced felt like it was happening to their own limb and all participants reported

459 that it felt like it was their own limb that was changing (irrespective of perspective). This area is

460 clearly ripe for future research, for example, to elucidate whether vividness of the illusion relates

461 to the degree of analgesia and whether or not ownership also occurs during incongruent VT

462 illusions.

463 Conclusions:

464 This study adds to the existing evidence suggesting that manipulation of body-relevant sensory

465 information has a modulatory effect on pain. Our results extend previous work by showing that 466 pain modulation by illusory resizing also occurs in knee OA and by clearly demonstrating that

467 the visual component of the congruent VT illusion is critical but requires multisensory input to

468 have an analgesic effect. Such results warrant replication in a larger sample, providing a greater

469 dosage to ascertain whether daily pain scores can be impacted. 


\section{Acknowledgements:}

471 We would like to thank Dr Valeria Bellan for her initial help in the piloting phase of this study.

472

473 


\section{$474 \quad$ Figure legends}

475 Figure 1. Experimental conditions and their statistical comparisons.

476 A. The eight experimental and control conditions. The red arrow indicates the direction of tactile

477 input provided. In the Congruent Visuotactile illusion, the tactile input directionally 'matched'

478 the visual manipulation (i.e., knee visually shrunk to look smaller, tactile push towards the knee

479 to 'match' visual input); in the Incongruent Visuotactile condition, the tactile input did not

480 directionally 'match' the visual manipulation (e.g., knee visually shrunk to look smaller, tactile

481 pull away from the knee, 'unmatched' to visual input). Photograph credit: Anne Graham.

482 B. Statistical comparisons. The grey shaded areas represent the control conditions for which the

483 most analgesic congruent visuotactile illusion was compared to for analysis purposes.

484 Figure 2. Pre-/post-condition pain scores comparing experimental conditions. Pain intensity was 485 rated on a $0-100$ NRS where $0=$ no pain at all and $100=$ worst pain imaginable. $*$ p $<0.05$; N.S. $=$ 486 non-significant

487 A. Mean pre- and post-condition pain scores ( \pm SEM) for comparisons between the Congruent 488 VT illusion and its components: vision only control, tactile only control. A significant Condition

$489 \mathrm{x}$ Time interaction was found; post-hoc comparisons showed that the congruent VT illusion 490 provided significant analgesia, while both component conditions did not.

491 B. Mean pre- and post-condition pain scores ( \pm SEM) for comparisons between the Congruent

492 VT illusion and the Incongruent VT Conditions. Separate repeated measures ANOVAs showed a 493 main effect of Time (pre-/post-) when the visual manipulation was identical (i.e., tactile input 494 differed) in Congruent and Incongruent conditions, but no effect when the tactile input was 495 identical (i.e., visual manipulation differed) in Congruent and Incongruent conditions. 
496 Figure 3. Mean pre- and post-illusion pain scores ( \pm SEM) over 10 repeated illusion trials. Pain 497 intensity was rated on a $0-100$ NRS where $0=$ no pain at all and $100=$ worst pain imaginable.

498 Planned comparisons performed between the first and tenth illusion trial, show that ten repeated 499 illusions significantly reduce both pre-illusion and post-illusion pain. ${ }^{*} \mathrm{p}<0.05$

500

501 
502

503

504

505

506

507

508

509

510

511

512

513

514

515

516

517

518

519

520

521

522

523

524

525

526

527

528

529

530

531

\section{References}

Abrams RA, and Christ SE. 2003. Motion onset captures attention. Psychol Sci 14:427-432.

Altman R, Asch E, Bloch D, Bole G, Borenstein D, Brandt K, Christy W, Cooke TD, Greenwald R, Hockberg M, and al e. 1986. Development of criteria for the classification and reporting of OA. Classification of OA of the knee. Diagnostic and Therapeutic Criteria Committee of the American Rheumatism Association. Arthritis Rheum 29:1039-1049.

Avillac M, Olivier E, Deneve S, Hamed SB, and Duhamel J-R. 2004. Multisensory integration in multiple reference frames in the posterior parietal cortex. Cognitive Processing 5:159166.

Bellan V, Gilpin HR, Stanton TR, Newport R, Gallace A, and Moseley GL. 2015. Untangling visual and proprioceptive contributions to hand localisation over time. Exp Brain Res 233:1689-1701.

Blanke O. 2012. Multisensory brain mechanisms of bodily self-consciousness. Nature Rev Neurosci 13:556.

Blanke O, Slater M, and Serino A. 2015. Behavioral, neural, and computational principles of bodily self-consciousness. Neuron 88:145-166.

Boesch E, Bellan V, Moseley GL, and Stanton TR. 2016. The effect of bodily illusions on clinical pain: a systematic review and meta-analysis. Pain 157:516-529.

Botan V, Fan S, Critchley H, and Ward J. 2018. Atypical susceptibility to the rubber hand illusion linked to sensory-localised vicarious pain perception. Consciousness and Cognition 60:62-71.

Bremmer F, Schlack A, Shah NJ, Zafiris O, Kubischik M, Hoffmann K, Zilles K, and Fink GR. 2001. Polymodal motion processing in posterior parietal and premotor cortex: a human fMRI study strongly implies equivalencies between humans and monkeys. Neuron 29:287-296.

Cardini F, Longo MR, and Haggard P. 2014. Vision of the body modulates somatosensory intracortical inhibition. Cereb Cortex 21:2014-2022.

Catley MJ, Tabor A, Wand BM, and Moseley GL. 2013. Assessing tactile acuity in rheumatology and musculoskeletal medicine - how reliable are two-point discrimination tasks at the neck, hand, back and foot? Rheumatology (Oxford) 52:1454-1461. 
532 Cohen J. 1969. Statistical power analyses for the behavioural sciences. New York: Academic 533 Press.

534 Cross M, Smith E, Hoy D, Nolte S, Ackerman I, Fransen M, Bridgett L, Williams S, Guillemin 535 F, Hill CL, Laslett LL, Jones G, Cicuttini F, Osborne R, Vos T, Buchbinder R, Woolf A, 536 and March L. 2014. The global burden of hip and knee osteoarthritis: estimates from the 537 Global Burden of Disease 2010 study. Ann Rheum Dis 73:1323-1330.

538 Dawon J, Fitzpatrick R, Carr A, and Murray D. 1998. Questionnaire on the perceptions of 539 patients about total hip replacemen. J Bone Joint Surg Br 80:63-69.

540 Farrar JT, Young JP, LaMoreaux L, Werth JL, and Poole RM. 2001. Clinical importance of $541 \quad$ changes in chronic pain intensity when measured on an 11-point numerical pain rating $542 \quad$ scale. Pain 94:149-158.

543 Faul F, Erdfelder E, Lang AG, and Buchner A. 2007. G*Power 3: A flexible statistical power 544 analysis program for the social, behavioural and biomedical sciences. Behaviour $545 \quad$ Research Methods 39:175-191.

546 Gallace A, Torta DM, Moseley GL, and Iannetti GD. 2011. The analgesic effect of crossing the $547 \quad$ arms. Pain 152:1418-1423.

548 Gilpin HR, Bellan V, Gallace A, and Moseley GL. 2014. Exploring the roles of body ownership, $549 \quad$ vision and virtual reality on heat pain threshold. Eur J Pain 18:900-901.

550 Gilpin HR, Moseley GL, Stanton TR, and Newport R. 2015. Evidence for distorted mental 551 representation of the hand in osteoarthritis. Rheumatology (Oxford) 54:678-682.

552 Giske L, Sandvik L, and Roe C. 2010. Comparison of daily and weekly retrospectively reported 553 pain intensity in patients with localized and generalized musculoskeletal pain. Eur J Pain $554 \quad 14: 959-965$.

555 Hannan MT, Felson DT, and Pincus T. 2000. Analysis of the discordance between radiographic $556 \quad$ changes and knee pain in osteoarthritis of the knee. J Rheumatology 27:1513-1517.

557 Holm S. 1979. A simple sequentially rejective multiple test procedure. Scand J Statistics 6:65$558 \quad 70$.

559 Kakigi R, and Watanabe S. 1996. Pain relief by various kinds of interference stimulation applied 560 to the peripheral skin in humans: pain-related brain potentials following $\mathrm{CO} 2$ laser 561 stimulation. J Peripher Nerv Syst 1:189-198. 
562 Lewis JS, and Schweinhardt P. 2012. Perceptions of the painful body: The relationship between 563 body perception disturbance, pain and tactile discrimination in complex regional pain $564 \quad$ syndrome Eur J Pain 16:1320-1330.

565 566 567 568 569 570 571 572 573 574 575 576
Longo MR, Betti V, Aglioti SM, and Haggard P. 2009. Visually induced analgesia: seeing the body reduces pain. $J$ Neurosci 29:1215-1230.

Longo MR, Iannetti GD, Mancini F, Driver J, and Haggard P. 2012. Linking pain and the body: Neural correlates of visually induced analgesia. J Neurosci 32:2601-2607.

Mancini F, Bauleo A, Cole J, Lui F, Porro CA, Haggard P, and Iannetti GD. 2014. Whole-body mapping of spatial acuity for pain and touch. Ann Neurol 75:917-924.

Mancini F, Longo MR, Kammers MPM, and Haggard P. 2011. Visual distortion of body size modulates pain perception. Psychol Sci 22:325-330.

Martini M, Perez-Marcos D, and Sanchez-Vives MV. 2014a. Author's reply to the commentary by Gilpin et al. Eur J Pain 19:143-144.

Martini M, Perez-Marcos D, and Sanchez-Vives MV. 2014b. Modulation of pain threshold by virtual body ownership. Eur J Pain 18:1040-1048.

McCabe CS, Cohen H, and Blake DR. 2007. Somaesthetic disturbances in fibromyalgia are exaggerated by sensory-motor conflict: implications for chronicity of the disease? . Rheumatology (Oxford) 46:1587-1592.

McCabe CS, Haigh RC, Halligan PW, and Blake DR. 2003. Distorting proprioception in chronic pain patients exacerbates sensory disturbances_-implications for pathology. Rheumatology (Oxford) 42:145.

Mohan R, Jensen KB, Petkova KI, Dey A, Barnsley N, Ingvar M, McAuley JH, and Moseley GL. 2012. No pain relief with the rubber hand illusion. PLoS ONE 7:e52400.

Moseley GL. 2005. Distorted body image in complex regional pain syndrome. Neurology $65: 773$.

Moseley GL, Gallace A, and Spence C. 2012. Bodily illusions in health and disease: physiological and clinical perspectives and the concept of a cortical 'body matrix'. Neurosci Biobehav Rev 36:34-46.

Moseley GL, and Gandevia SC. 2005. Sensory-motor incongruence and reports of 'pain'. Rheumatology 44:1083-1085. 
592 Moseley GL, Parsons LM, and Spence C. 2008. Visual distortion of a limb modulates the pain $593 \quad$ and swelling evoked by movement. Curr Biol 18:R1047-R1048.

594 Nguyen MHV, Yeasted RE, and Schnitzer TJ. 2014. WOMAC Pain Score reflects preceding 595 daily pain ratings in knee osteoarthritis interventional randomized clinical trials. 2014 596 ACR/ARHP Annual Meeting. Boston.

597 Nierula B, Martini M, Matamala-Gomez M, Slater M, and Sanchez-Vives MV. 2017. Seeing an 598 Embodied Virtual Hand is Analgesic Contingent on Colocation. J Pain 18:645-655. 599 Nishigami T, Mibu A, Tanaka K, Yamashita Y, Yamada E, Wand BM, Catley MJ, Stanton TR, 600 and Moseley GL. 2017. Development and psychometric properties of the knee-specific 601 body-perception questionnaire in people with knee osteoarthritis: The Fremantle Knee $602 \quad$ Awareness Questionnaire. PLoS ONE 12:e0179225.

603 Penfield W, and Boldrey E. 1937. Somatic motor and sensory representation in the cerebral 604 cortex of man as studied by electrical stimulation. Brain 60:389-443.

605 Perro S, Marty M, Legout V, Moyse D, Henrotin Y, and Rozenberg S. 2011. Ecological or 606 recalled assessments in chronic musculoskeletal pain? A comparative study of 607 prospective and recalled pain assessments in low back pain and lower limb painful 608 osteoarthritis. Pain Med 12:427-436.

609 Pozeg P, Galli G, and Blanke O. 2015. Those are your legs: The effect of visuo-spatial viewpoint 610 on visuo-tactile integration and body ownership. Front Psychol 6:1749.

611 Preston C, Kuper-Smith BJ, and Ehrsson HH. 2015. Owning the body in the mirror: The effect of 612 visual perspective and mirror view on the full-body illusion. Sci Rep 5:18345.

613 Preston C, and Newport R. 2011. Analgesic effects of multisensory illusions in osteoarthritis. $614 \quad$ Rheumatology 50:2314-2315.

615 Ratcliffe N, and Newport R. 2017. The effect of visual, spatial and temporal manipulations on 616 embodiment and action. Front Hum Neurosci 4:227.

617 Rohde M, Di Luca M, and Ernst MO. 2011. The rubber hand illusion: Feeling of ownership and 618 proprioceptive drift do not go hand in hand. PLOS ONE 6:e21659.

619 Salaffi F, Stancati A, Silvestri CA, Ciapetti A, and Grassi W. 2004. Minimal clinically important 620 changes in chronic musculoskeletal pain intensity measured on a numerical rating scale. $621 \quad$ Eur J Pain 8:283-291. 
622 Schaefer M, Flor H, Heize HJ, and Rotte M. 2007. Morphing the body: illusory feeling of an 623 elongated arm affects somatosensory homunculus. Neuroimage 36:700-705.

624 Siedlecka M, Klimza A, Lukowska M, and Wierzchon M. 2014. Rubber hand illusion reduces 625 discomfort caused by cold stimulus. PLOS ONE 9:e109909.

626 Stein BE, Standford TR, and Rowland BA. 2014. Development of multisensory integration from 627 the perspective of the individual neuron. Nature Rev Neurosci 15:520-535.

628 Stein BE, and Stanford TR. 2008. Multisensory integration: current issues from the perspective 629 of the single neuron. Nature Rev Neurosci 9:255-266.

630 van Elk M, Forget J, and Blanke O. 2013. The effect of limb crossing and limb congruency on 631 multisensory integration in peripersonal space for the upper and lower extremities.

632 Consciousness and Cognition 22:545-555.

633 Wylde V, Hewlett S, Learmonth ID, and Dieppe P. 2011. Persistent pain after joint replacement: 634 prevalence, sensory qualities, and postoperative determinants. Pain 152:566-572.

635 


\section{Figure 1}

Experimental conditions and their statistical comparisons.

A. The 8 experimental and control conditions. The red arrow indicates the direction of tactile input provided. In the Congruent Visuotactile illusion, the tactile input directionally 'matched' the visual manipulation (i.e., knee visually shrunk to look smaller, tactile push towards the knee to 'match' visual input); in the Incongruent Visuotactile illusion, the tactile input did not directionally 'match' the visual manipulation (e.g., knee visually shrunk to look smaller, tactile pull away from the knee, 'unmatched' to visual input). Photograph credit: Anne Graham. B. Statistical comparisons. The grey shaded areas represent the control conditions for which the most analgesic congruent visuotactile illusion was compared to for analysis purposes.

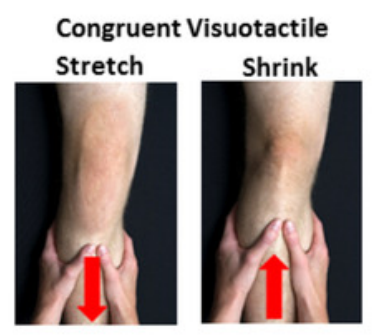

Incongruent Visuotactile control Stretch

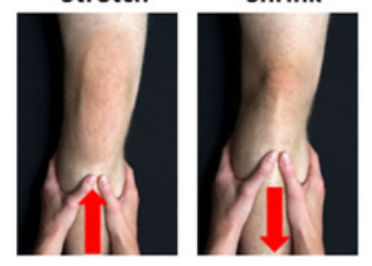

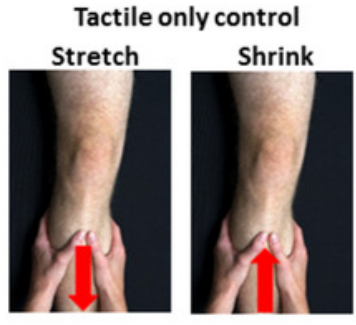

Vision only control

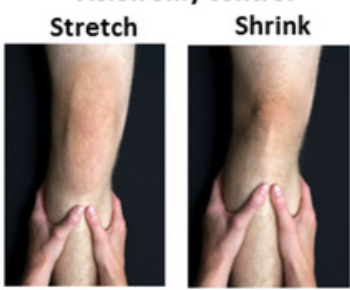

B.

\begin{tabular}{|l|l|l|}
\hline & \multicolumn{2}{|c|}{ Most analgesic illusion } \\
\hline Control Condition & Congruent stretch & \multicolumn{1}{c|}{ Congruent shrink } \\
\hline Tactile stretch & & \\
\hline Tactile shrink & & \\
\hline Visual stretch & & \\
\hline Visual shrink & & \\
\hline Incongruent stretch & $\begin{array}{l}\text { Controls for vision; } \\
\text { Tactile input differs }\end{array}$ & $\begin{array}{l}\text { Controls for tactile; } \\
\text { Visual input differs }\end{array}$ \\
\hline Incongruent shrink & $\begin{array}{l}\text { Controls for tactile; } \\
\text { Visual input differs }\end{array}$ & $\begin{array}{l}\text { Controls for vision; } \\
\text { Tactile input differs }\end{array}$ \\
\hline
\end{tabular}




\section{Figure 2 (on next page)}

\section{Pre-/post-condition pain scores comparing experimental conditions.}

Pain intensity was rated on a $0-100$ NRS where $0=$ no pain at all and $100=$ worst pain imaginable. * $\mathrm{p}<0.05 ;$ N.S. $=$ non-significant

A. Mean pre- and post-condition pain scores ( \pm SEM) for comparisons between the Congruent VT illusion and its components: vision only control, tactile only control. A significant Condition $\mathrm{x}$ Time interaction was found; post-hoc comparisons showed that the congruent VT illusion provided significant analgesia, while both component conditions did not.

B. Mean pre- and post-condition pain scores ( \pm SEM) for comparisons between the Congruent VT illusion and the Incongruent VT Conditions. Separate repeated measures ANOVAs showed a main effect of Time (pre-/post-) when the visual manipulation was identical (i.e., tactile input differed) in Congruent and Incongruent conditions, but no effect when the tactile input was identical (i.e., visual manipulation differed) in Congruent and Incongruent conditions. 

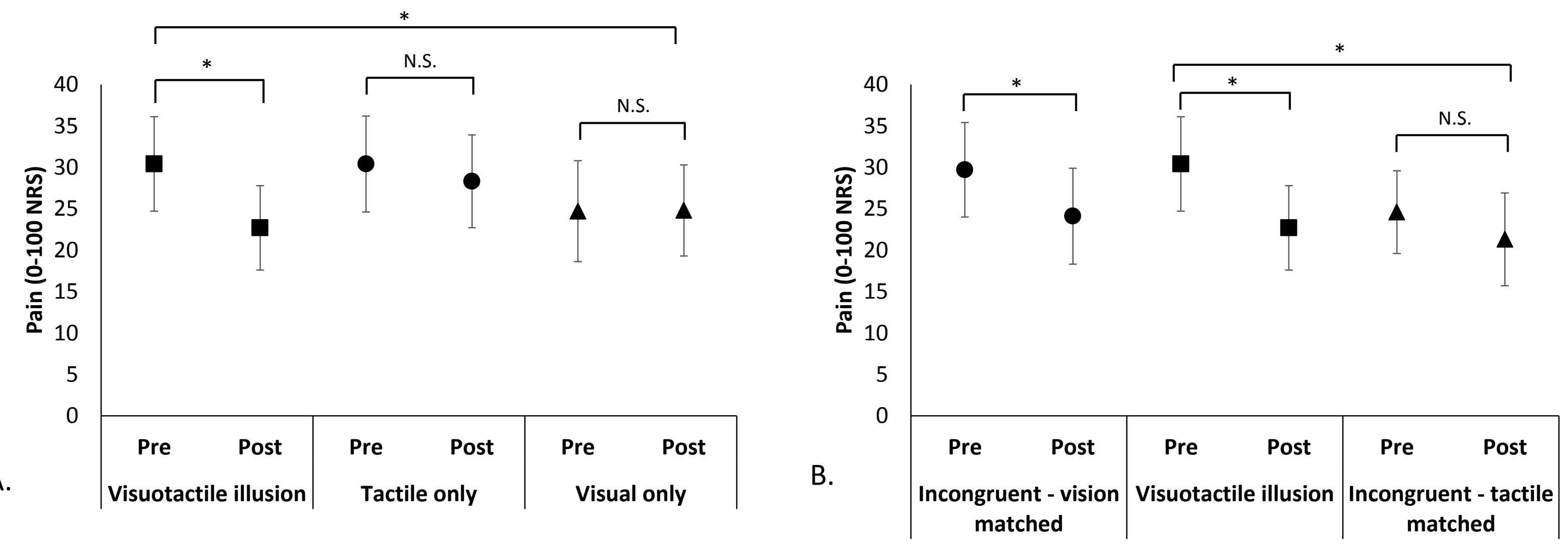


\section{Figure 3 (on next page)}

Pre- and post-illusion pain scores over 10 repeated illusions.

Planned comparisons performed between illusion 1 and 10, show that 10 repeated illusions significantly reduce both pre-illusion and post-illusion pain. $* p<0.05$ 


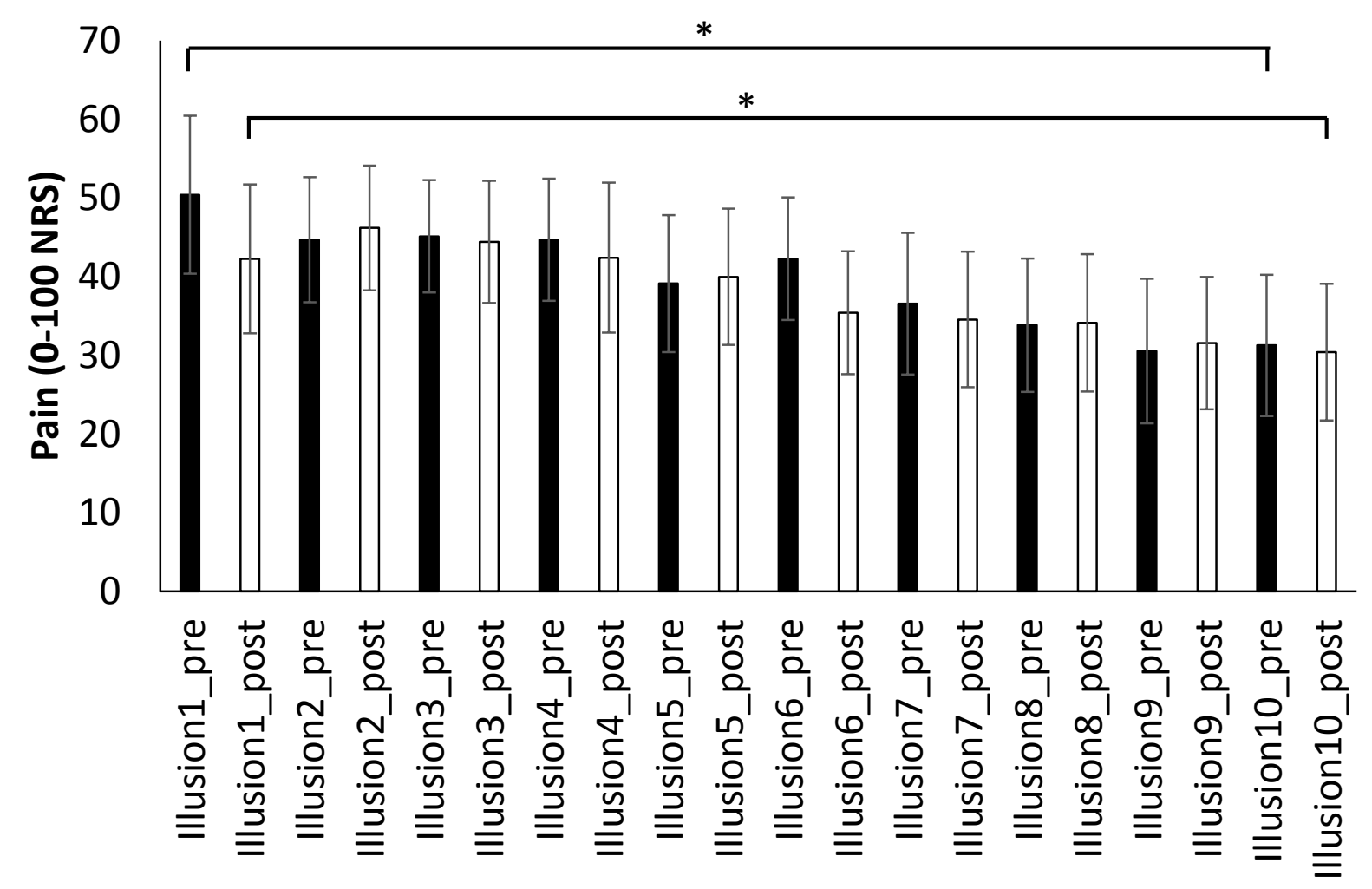




\section{Table $\mathbf{1}$ (on next page)}

Participant demographic and testing session outcomes.

All pain outcomes measured using a 101-point NRS. Oxford knee scores range from 0-48 where higher values indicate less disability. Knee awareness/perception was evaluated using a modified version of the Fremantle Knee Awareness Questionnaire (FreKAQ); scores range from 0-36 with higher scores reflecting less knee awareness (Nishigami et al. 2017). Perceived knee size was evaluated using established methodology (Gilpin et al. 2015): a picture of a participants' knee was altered in size; participants indicated when the viewed image appeared to be the correct size of their knee. 


\begin{tabular}{|c|c|}
\hline & Mean (SD) \\
\hline \multicolumn{2}{|l|}{ Demographics } \\
\hline Age (years) & $67.3(9.9)$ \\
\hline Gender (count) & 9 female \\
\hline Height $(\mathrm{cm})$ & $167.2(11.2)$ \\
\hline Weight $(\mathrm{kg})$ & $82.7(16.3)$ \\
\hline Bilateral painful knee OA (count) & 6 \\
\hline History of knee pain tested knee (years) & $16.5(14.3)$ \\
\hline History of knee pain untested knee (years) & $7.0(5.4)$ \\
\hline Average baseline knee pain (past $48 \mathrm{hrs}$ ) & $48.0(24.3)$ \\
\hline Maximum knee pain (past $48 \mathrm{hrs}$ ) & $66.3(28.6)$ \\
\hline Minimum knee pain (past 48 hrs) & $6.3(10.9)$ \\
\hline Oxford knee score & $24.1(8.1)$ \\
\hline Knee awareness/perception (FreKAQ) & $14.0(8.4)$ \\
\hline Perceived knee size ( $\%$ of true size) & $104.0(0.05)$ \\
\hline \multicolumn{2}{|l|}{ Session one } \\
\hline $\begin{array}{l}\text { Visuotactile illusion resulting in the most } \\
\text { analgesia (count) }\end{array}$ & stretch -7 ; equivocal -2 ; shrink -3 \\
\hline 'Best' illusion (visuotactile or visual only) & visuotactile -9 ; visual -3 \\
\hline \multicolumn{2}{|l|}{ Sustained illusion: } \\
\hline Post-illusion pain (directly after) & $28.5(17.0)$ \\
\hline Sustained: post-illusion pain (180 seconds) & $26.4(18.9)$ \\
\hline \multicolumn{2}{|l|}{ Session two } \\
\hline \multicolumn{2}{|l|}{ Repeated illusions: } \\
\hline Pre-illusion 1 pain & $31.7(12.9)$ \\
\hline Pre-illusion 10 pain & $21.7(17.5)$ \\
\hline Post-illusion 1 pain & $23.3(8.8)$ \\
\hline Post illusion 10 pain & $17.2(16.6)$ \\
\hline \multicolumn{2}{|l|}{ Session three } \\
\hline \multicolumn{2}{|l|}{ Sustained illusion: } \\
\hline Post-illusion pain (directly after) & $27.4(15.5)$ \\
\hline Sustained: post-illusion pain (180 seconds) & $28.4(17.7)$ \\
\hline \multicolumn{2}{|l|}{ Repeated illusions: } \\
\hline Pre-illusion 1 pain & $50.4(24.6)$ \\
\hline Pre-illusion 10 pain & $31.3(22.0)$ \\
\hline Post-illusion 1 pain & $42.3(23.1)$ \\
\hline Post illusion 10 pain & $30.4(21.3)$ \\
\hline \multicolumn{2}{|l|}{ Daily pain scores } \\
\hline 48 hours after session 2 & $45.1(16.8)$ \\
\hline 48 hours before session 3 & $58.1(25.2)$ \\
\hline
\end{tabular}

1 Table 1. Participant demographic and testing session outcomes. All pain outcomes measured 2 using a 101-point NRS. Oxford knee scores range from 0-48 where higher values indicate less 3 disability. Knee awareness/perception was evaluated using a modified version of the Fremantle 4 Knee Awareness Questionnaire (FreKAQ); scores range from 0-36 with higher scores reflecting 5 less knee awareness (Nishigami et al. 2017). Perceived knee size was evaluated using established 
6 methodology (Gilpin et al. 2015): a picture of a participants' knee was altered in size;

7 participants indicated when the viewed image appeared to be the correct size of their knee. 